Naoki Osada · Jun Kusuda • Yutaka Suzuki

Sumio Sugano • Katsuyuki Hashimoto

\title{
Sequence analysis, gene expression, and chromosomal assignment of mouse Borg4 gene and its human orthologue
}

\begin{abstract}
The Borg (binder of Rho GTPases) family proteins interact with $\mathrm{CDC} 42$ and $\mathrm{TC} 10$ in a guanosine triphosphate (GTP)-dependent manner. We have isolated a full-length cDNA of the mouse Borg4 gene, which is a member of this family. Sequence analysis revealed that this gene encoded a putative 349 -amino acid protein. By reverse transcription - coupled polymerase chain reaction (RTPCR) analysis, we observed that Borg4 was expressed ubiquitously in adult tissues. Additionally, we determined the entire cDNA sequence of the putative human Borg4 orthologue. By fluorescence in situ hybridization, mouse Borg4 and the putative human orthologue have been assigned to mouse chromosome $11 \mathrm{E}$ and human chromosome 17q24-25, which has been described as syntenic to the mouse region.
\end{abstract}

Key words Cell motility $\cdot$ Binder of Rho GTPase $\cdot$ CDC42 TC10 - Borg4 $\cdot$ Full-length cDNA · Fluorescence in situ hybridization

N. Osada $(\bowtie) \cdot$ J. Kusuda $\cdot$ K. Hashimoto

Division of Genetic Resources, National Institute of Infectious

Diseases, 1-23-1 Toyama-cho, Shinjuku-ku, Tokyo 162-8640, Japan

Tel. +81-3-5258-1111; Fax +81-3-5285-1181

e-mail: osada@nih.go.jp

N. Osada

Department of Biological Science, Graduate School of Science, The

University of Tokyo, Tokyo, Japan

Y. Suzuki ${ }^{1} \cdot$ S. Sugano

Department of Virology, Institute of Medical Science, The

University of Tokyo, Tokyo, Japan

Present address:

${ }^{1}$ Genome Science Center, Institute of Physical and Chemical

Research (RIKEN), Saitama, Japan

\section{Introduction}

Cell motility and morphogenesis are considerably complex phenomena, and the molecular mechanisms are still under investigation. Because an extremely large number of genes collaborate and there is cross-talk between them in these phenomena, it is essential to completely identify the unknown genes involved in the processes. The Rho family of guanosine triphosphatases (GTPases) is known to play important roles in cytoskeletal modeling (Aelst and D'SouzaSchorey 1997: Kjoller and Hal 1999), and, interestingly, it is reported that a member of the Rho protein family, named TC10, promotes nerve elongation in neuronal cells (Tanabe et al. 2000). Recently, a new protein family, named Borg, which interacts with $\mathrm{Cdc} 42$ or TC10, has been identified (Joberty et al. 1999). The proteins of this family have been shown to share the CRIB (Cdc42, Rac interactive binding) domain and specific domains named BH (Borg homology) 1 , 2 , and 3. The CRIB domain is an amino acid motif (consensus sequence: I-S-X-P- $\left.(\mathrm{X})_{2-4}-\mathrm{F}-\mathrm{X}-\mathrm{H}-\mathrm{X}-\mathrm{X}-\mathrm{H}-\mathrm{V}-\mathrm{G}\right)$ which defines candidates for effector proteins of Cdc42 and Rac GTPases in a GTP-dependent manner (Berbelo et al. 1995). The ectopic expression of Borg1, 2, and 3 induces the loss of actin stress fibers, suggesting that the Borgs inhibit the Rho function. The Borg family, Borg1 to Borg5 (Borg5 was previously known as MSE55) (Bahou et al. 1992) cDNAs were isolated using a two-hybrid screen and expressed sequence tags (EST) database and were revealed to contain several highly conserved BH1, 2, and 3 domains, as well as CRIB. As for Borg4, however, only CRIB and BH1 could be demonstrated, because of the partial cDNA isolates (Joberty et al. 1999).

Here we report the cloning of the full-length mouse Borg4 and its human orthologue cDNAs. Borg4 shares, in common with the other Borg family members, highly conserved CRIB, BH1, and BH3 domains, and a less well conserved $\mathrm{BH} 2$ domain. We further showed that Borg4 was expressed ubiquitously at low levels in adult mouse tissues, and mapped mouse Borg 4 to mouse chromosome 11E and the human orthologue to human chromosome 17q24-25 by fluorescence in situ hybridization (FISH). 


\section{Materials and methods}

\section{DNA sequencing}

The mouse Borg4 cDNA clone (MNCb-0220; DDBJ/ EMBL/GenBank accession number AB035088; the 5'part of the sequence was deposited as accession number AU035226) and the putative human orthologue clone (KAIA1777; accession number AB042237) were derived from a mouse brain and a human adult ileum mucosa cDNA library made by the oligo-capping method (Suzuki et al. 1997; Suzuki et al. 2000). The method allowed us to replace the cap structure of mRNA with synthetic oligonucleotides (5'-oligo), which could then serve as the sequence tag for the mRNA start site. Using the oligo-capped mRNAs and oligo-dT primer, we were able to amplify full-length cDNAs at a high frequency. The insert was sequenced on an ABI 310 automated sequencer (PerkinElmer, Norwalk, CT, USA) by the primer walking method.

Reverse transcription - coupled polymerase chain reaction (RT-PCR)

Primers used for RT-PCR were designed to amplify the 362-bp fragment (bases 2691-3052 of Borg4 cDNA). The primers were 5'-GGGGTGTGAGCTACGCTTT-3' and 5'-CCCAGCACAGGAAAGTAATGA-3'. The templates of the mouse total mRNA were purchased from OriGene (Rockville, MD, USA) and treated with RNase-free DNase-I (Takara, Kyoto, Japan) before amplification. One microliter of total mRNA was amplified, using the One Step RNA PCR Kit (Takara). Temperature and time schedules were: 40 cycles of $94^{\circ} \mathrm{C}$ for $30 \mathrm{~s}, 60^{\circ} \mathrm{C}$ for $30 \mathrm{~s}$, and $72^{\circ} \mathrm{C}$ for $90 \mathrm{~s}$. PCR products were separated on $1.5 \%$ agarose gel with a 100-bp ladder DNA marker (GIBCO-BRL, Gaithersburg, MD, USA).

Chromosomal mapping by fluorescence in situ hybridization (FISH)

FISH was performed as described previously (Hirai et al. 1996). After overnight hybridization with biotin-labeled Borg 4 cDNA, the slides were washed in $50 \%$ formamide $/ 2$ $\times$ standard saline citrate (SSC) at $42^{\circ} \mathrm{C}$ for $15 \mathrm{~min}$, followed by a wash in $1 \times \mathrm{SSC}$ at room temperature for $15 \mathrm{~min}$. The slides were then blocked with $4 \%$ bovine serum albumin $/ 4$ $\times \mathrm{SSC}$ at $37^{\circ} \mathrm{C}$ for $30 \mathrm{~min}$. Signal amplification was achieved using rabbit anti-biotin (ENZO, New York, NY, USA), fluorescein-labeled goat anti-rabbit IgG (ENZO), and Cy2-labeled donkey anti-goat IgG (Amersham Pharmacia, Uppsala, Sweden). The chromosomes were counterstained with propidium iodide. Slides were examined with a fluorescence microscope (BX60; Olympus, Tokyo, Japan) equipped with an IB filter. For mouse, the same metaphase spreads were also viewed with a UV filter for G-band analysis.

\section{Results and discussion}

We have isolated full-length cDNA clones from a mouse brain cDNA library constructed by Sugano et al., using the oligo-capping method, as described previously (Suzuki et al. 1997). Among a number of complete nucleotide (nt) sequences of cDNA clones sequenced, we found the clone MNCb-0220, which completely matched the partial mouse Borg4 nucleotide sequence (accession number AF165114). The insert length of the cDNA clone is $3420 \mathrm{bp}$ and the deduced amino acid has 349 residues (corresponding to $\mathrm{nt}$ 691-1737), with a predicted mass of $37.9 \mathrm{kDa}$ and a hypothetical pI of 5.20. Compared with the other family member proteins, the mouse Borg4 protein was shown to contained highly conserved CRIB, BH1, and BH3 domains, and a less conserved $\mathrm{BH} 2$ domain (Fig. 1A).

By further database searching, we found a candidate cDNA sequence for the human Borg4 homologue deposited in GenBank as CEP4 (cdc42 effector protein 4; accession number AF099664). However, the CEP4 nucleotide sequence in the database was incomplete in the untranslated region; therefore, we searched for a full-length Borg4 cDNA clone in a full-length enriched cDNA library from human adult ileum mucosa (Suzuki et al. 2000) and found the clone KAIA1777. The insert length of KAIA1777 was $2732 \mathrm{bp}$ and the coding sequence shared $80 \%$ identity with that of mouse Borg4. The deduced amino acid sequence of human Borg4, as designated here, and mouse Borg4 are aligned in Fig. 1B. The sequences of the Nterminal region are well conserved, whereas those of the Cterminal flanking region vary greatly (total identity, $77 \%$ ). All three Borg homology domains are highly conserved in the two proteins, suggesting the functional significance of these domains (Fig. 1B, shown by rectangles). In the 5'untranslated region (UTR), only the 70-bp sequence juxtaposing the translation start site showed high homology, but it was reduced in the upstream region, suggesting that this 70-bp segment possessed some functional element. The 3'UTR of KAIA1777, however, was quite dissimilar to mouse Borg4 and contained 173bp of the medium reiteration frequency repeat (MER53). To confirm that this dissimilarity between mouse/human Borg4 was not due to the chimeric clones, polymerase chain reaction (PCR) analysis was performed, using a primer set derived from two regions with different similarity scores. The effective amplification of the DNA fragment from the human genomic boundary region allowed us to suppose that the discordance might be responsible for alternative exon or polyA signal choices, and affect the transcriptional regulation.

It is reported that Borg family genes are expressed in distinct patterns (Bahou et al. 1992; Joberty et al. 1999). However, Joberty et al. failed to show the expression pattern of Borg4 and suggested that it may be expressed only in fetal tissues. Because we had isolated the mouse Borg4 fulllength cDNA from an adult mouse brain cDNA library, we performed northern blot analysis, using the cDNA as a probe to confirm its expression. But no signal for the Borg4 transcripts was observed in any tissues (data not shown). 
Fig. 1. A Alignment of the Borg family proteins for the CRIB, $\mathrm{BH} 1, \mathrm{BH} 2$, and $\mathrm{BH} 3$ domains. Borg3 lacks the conserved $\mathrm{BH} 2$ domain. B Alignment of KAIA1777 protein (putative human Borg4 homologue) and mouse Borg4. Identities are indicated by black background, and similar residues are shadowed
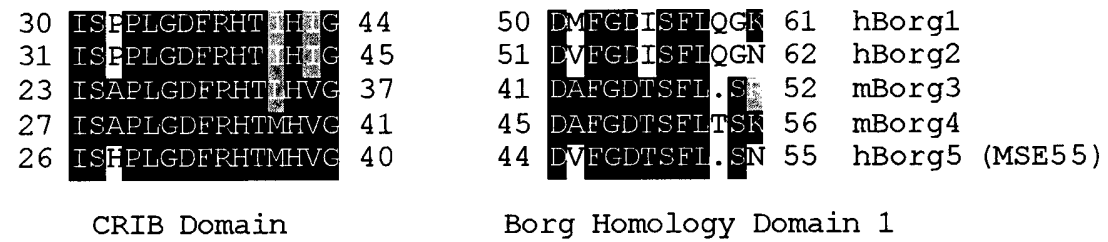

Borg Homology Domain 1

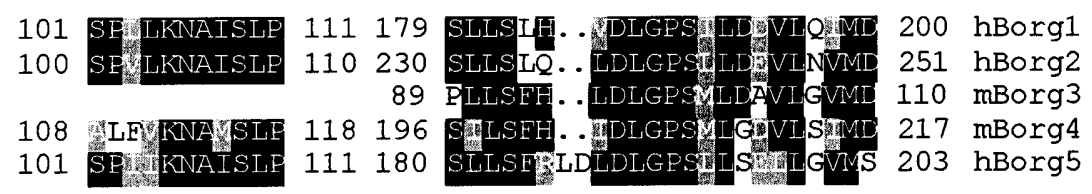

A Borg Homology Domain 2

Borg Homology Domain 3

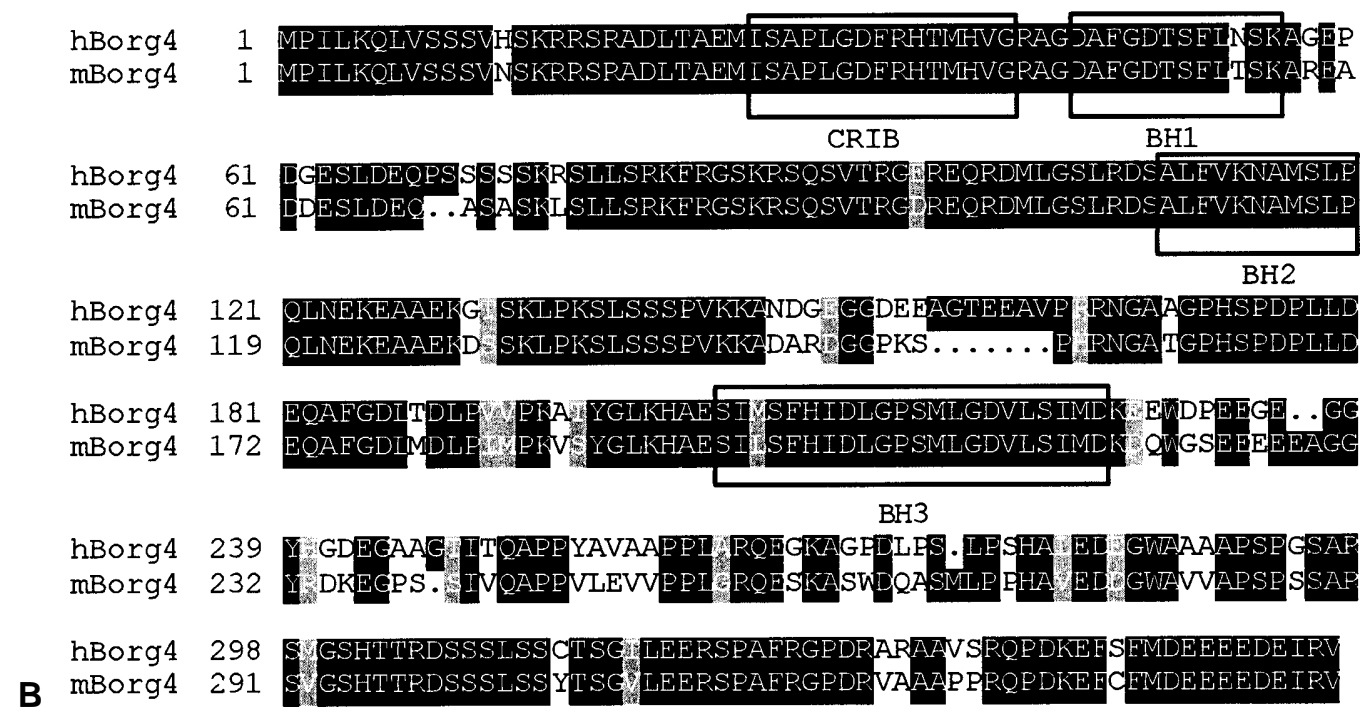

Subsequently, we used an RT-PCR method to assay its expression. The result showed that Borg4 was expressed ubiquitously in adult tissues (Fig. 2), unlike the nonuniformly expressed other family proteins. Considering the result of northern blot analysis, mouse Borg4 may be expressed at quite low levels.

Although a working draft of the human genome sequence has been available in the NCBI database, we found no genomic sequence preferably matched to the KAIA1777 and MNCb-0220 clones. To determine the chromosomal localization of Borg4, FISH was performed, using the MNCb-0220 and KAIA1777 clones as probes. For KAIA1777, we detected FISH signals on human chromosome 17q24-25 (Fig. 3A), and this result was confirmed by PCR analysis of human and rodent somatic cell hybrid panels (data not shown). With MNCb-0220, specific signals were observed in the mouse chromosome 11E region (Fig. $3 \mathrm{~B}, \mathrm{C})$. To confirm the chromosome identification, a painting probe of mouse chromosome 11 was hybridized to the same metaphase spreads after signal detection (data not

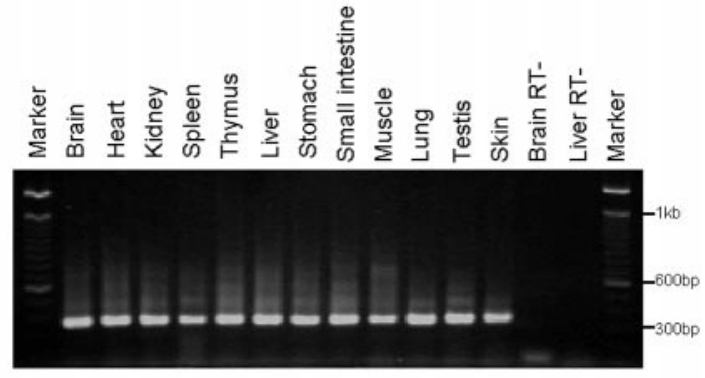

Fig. 2. Tissue distribution analysis of mouse Borg4 transcript, using reverse transcription-coupled polymerase chain reaction (RT-PCR). The 12 tissues examined and the controls (- reverse transcriptase $R T$ ) are indicated above each lane

shown). Human chromosome 17q24-25 and mouse chromosome 11E have been described as syntenic regions (Carver and Stubbs 1997). Despite some dissimilarity in the nucleotide sequences, the chromosomal localization supports the idea that CEP4 is the orthologue of mouse Borg4. 

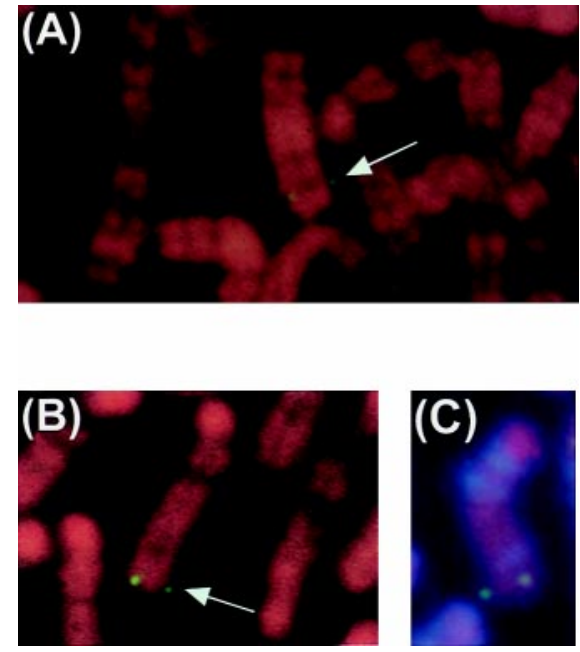

Fig. 3A-C. Chromosomal mapping of human (KAIA1777) and mouse (MNCb-0220) Borg4 genes by fluorescence in situ hybridization (FISH). A Doublet FISH signals (arrow) are observed on human chromosome 17q24-25. B Metaphase, showing doublet on mouse chromosome 11 at region 11E (arrow). C Another metaphase is viewed with a UV filter for G-band analysis

\section{References}

Aelst LV, D'Souza-Schorey C (1997) Rho GTPases and signaling networks. Genes Dev 11:2295-2322
Bahou WF, Campbell AD, Wicha MS (1992) cDNA cloning and molecular characterization of MSE55, a novel human serum constituent protein that displays bone marrow stromal/endothelial cell-specific expression. J Biol Chem 267:13986-13992

Berbelo PD, Drechsel D, Hall A (1995) A conserved binding motif defines numerous candidate target proteins for both $\mathrm{Cdc} 42$ and Rac GTPase. J Biol Chem 270:29071-29074

Carver EA, Stubbs L (1997) Zooming in on the human-mouse comparative map: genome conservation re-examined on a highresolution scale. Genome Res 7:1123-1137

Hirai M, Kusuda J, Hashimoto K (1996) Assignment of human ADP ribosylation factor (ARF) genes $A R F 1$ and $A R F 3$ to chromosomes $1 \mathrm{q} 42$ and 12q13, respectively. Genomics 34:263-265

Joberty G, Perlungher RR, Macara G (1999) The Borgs, a new family of Cdc42 and TC10 GTPase-interacting proteins. Mol Cell Biol 19:6585-6597

Kjoller L, Hal A (1999) Signaling to Rho GTPases. Exp Cell Res 253:166-179

Suzuki Y, Yoshimoto-Nakagawa K, Maruyama K, Suyama A, Sugano S (1997) Construction and characterization of a full-enriched length and a 5'-end-enriched cDNA library. Gene 200:149-156

Suzuki Y, Ishihara D, Sasaki M, Nakagawa H, Hata H, Tsunoda T, Watanabe M, Komatsu T, Ota T, Isogai T, Suyama A, Sugano S (2000) Statistical analysis of the 5' untranslated region of human mRNA using "oligo-capped" cDNA libraries. Genomics 64:286-297

Tanabe K, Tachibana T, Yamashita T, Che YH, Yoneda Y, Ohci T, Tohyama M, Yoshikawa H, Kiyama H (2000) The small GTPbinding protein TC10 promotes nerve elongation in neuronal cells and its expression is induced during nerve regeneration in rats. $\mathbf{J}$ Neurosci 20:4138-4144 